\title{
Prognostic Factor Analysis for Management of Chronic Neck Pain : Can We Predict the Severity of Neck Pain with Lateral Cervical Curvature?
}

\author{
Han Yu Seong, M.D., Moon Kyu Lee, M.D., ${ }^{2}$ Sang Ryong Jeon, M.D., Ph.D., ${ }^{3}$ Sung Woo Roh, M.D., Ph.D., ${ }^{3}$ \\ Seung Chul Rhim, M.D., Ph.D., Jin Hoon Park, M.D., Ph.D. ${ }^{4}$ \\ Department of Neurological Surgery, Bumin Hospital Seoul, Seoul, Korea \\ Departments of Neurology, ${ }^{2}$ Neurological Surgery, Gangneung Asan Hospital, University of Ulsan College of Medicine, Gangneung, Korea \\ Department of Neurological Surgery, ${ }^{3}$ Asan Medical Center, University of Ulsan College of Medicine, Seoul, Korea
}

Objective : Although little is known about its origins, neck pain may be related to several associated anatomical pathologies. We aimed to characterize the incidence and features of chronic neck pain and analyze the relationship between neck pain severity and its affecting factors.

Methods : Between March 2012 and July 2013, we studied 216 patients with chronic neck pain. Initially, combined tramadol (37.5 $\mathrm{mg}$ ) plus acetaminophen $(325 \mathrm{mg}$ ) was administered orally twice daily (b.i.d.) to all patients over a 2-week period. After two weeks, patients were evaluated for neck pain during an outpatient clinic visit. If the numeric rating scale of the patient had not decreased to 5 or lower, a cervical medial branch block (MBB) was recommended after double-dosed previous medication trial. We classified all patients into two groups (mild vs. severe neck pain group), based on medication efficacy. Logistic regression tests were used to evaluate the factors associated with neck pain severity.

Results : A total of 198 patients were included in the analyses, due to follow-up loss in 18 patients. While medication was successful in reducing pain in $68.2 \%$ patients with chronic neck pain, the remaining patients required cervical MBB. Lateral cervical curvature, such as a straight or sigmoid type curve, was found to be significantly associated with the severity of neck pain.

Conclusion : We managed chronic neck pain with a simple pharmacological management protocol followed by MBB. We should keep in mind that it may be difficult to manage the patient with straight or sigmoid lateral curvature only with oral medication.

Key Words : Neck pain · Cervical spine · Lateral curvature · Sigmoid curvature · Medial branch block.

- Received : October 6, 2015 •Revised : April 25, 2016 •Accepted : August 5, 2016

- Address for reprints : Jin Hoon Park, M.D., Ph.D.

Department of Neurological Surgery, Gangneung Asan Hospital, University of Ulsan College of Medicine, 38 Bangdong-gil, Sacheon, Gangneung 25440, Korea Tel : +82-33-610-3260, Fax : +82-33-610-4960, E-mail : grandblue@gnah.co.kr

This is an Open Access article distributed under the terms of the Creative Commons Attribution Non-Commercial License (http://creativecommons.org/licenses/by-nc/4.0) which permits unrestricted non-commercial use, distribution, and reproduction in any medium, provided the original work is properly cited. 


\section{INTRODUCTION}

Chronic neck pain is a common clinical symptom, affecting $50-75 \%$ of people for 1 year or longer after the initial episode $\mathrm{f}^{6,10-12,14,25,35)}$. The annual prevalence of neck pain has been reported to range from $30 \%$ to $50 \%{ }^{18,23,32)}$. Although little is known concerning the etiology of neck pain, possible pathogenic factors include pathological changes in spinal discs, facet joints, ligaments, fascia, muscles, nerve root, or dura. The facet joint has been reported to be one of the most common causative factors underlying chronic pain, based on the association between facet joint and neck pain and/or referred pain in the head and shoulders. In addition, evidence supporting the existence of cervical facet joint pain has been reported under conditions of controlled diagnostic pain block ${ }^{16,42)}$. For the management of cervical facet joint pain, previous studies have shown that medial branch block (MBB) and radio frequency neurotomy were effective interventions for intractable pain $^{2,3,28,30)}$. Neck pain controlled with MBB is generally regarded as more severe than pharmacologically controllable neck pain. In the present study, we evaluated the incidence and features of chronic neck pain in a single center. In addition, we analyzed the affecting factors for the severity of chronic neck pain.

\section{MATERIALS AND METHODS}

Two hundred thirty three patients who visited our outpatient clinic between March 2012 and July 2013, with a complaint of neck pain (duration $>3$ months with a numeric rating scale [NRS] of $>5$ ) were enrolled in this study. We prospectively managed those patients with same protocol and collect data under informed consent of all patients. Exclusion criteria included patients with radiculopathy, myelopathy, previous cervical spinal surgery, uncontrolled acute or chronic severe medical illness that could interfere with the interpretations of the outcome assessments, psychiatric disorders, difficulty with communication, or women who were pregnant or lactating. Seventeen patients were excluded from the study, resulting in a study population of 216 patients. Initially, all patients were prescribed a combination of oral tramadol (37.5 mg) and acetaminophen (325 mg) tablets, twice daily (b.i.d.), over a 2 -week period. At the conclusion of the drug treatment peri- od, patients returned to the outpatient clinic and were evaluated for changes in their neck pain. If the NRS of the patient had decreased to a score $<5$ or to $50 \%$ of the initial NRS, we prescribed a second round of the above-mentioned medication and advised patient to take medication as needed for pain. If the patient reported sustained neck pain, including an NRS score $>5$ or pain reduction less than $50 \%$, we increased the combined oral dosage of tramadol (75 mg) and acetaminophen $(650 \mathrm{mg})$ b.i.d. for an additional 2 weeks. Following the second course of medication, all patients returned to the outpatient clinic for pain evaluation. Same protocol in patient with insufficient pain reduction was used with double-dose medication. Cervical MBB was recommended for all patients failing to show significant pain reduction as described above.

All patients were advised that if side effects such as nausea or dizziness developed, the medication would be changed to a combination of oral acetaminophen (250 mg), ibuprofen (200 $\mathrm{mg})$, and codeine phosphate $(10 \mathrm{mg}$ ) three times daily (t.i.d.). If this medication regimen was not well tolerated, cervical MBB was also recommended. Treatment protocols are shown in Fig. 1.

We classified all patients into four groups according to the location of pain. Patients with lower occipital and neck pain were classified as Type I; patients with only neck pain were classified as Type II; patients with neck and upper shoulder pain were classified as Type III; and patients with lower occipital, neck, and shoulder pain were classified as Type IV (Fig. 2). According to previous reports, a dysfunctional cervical facet

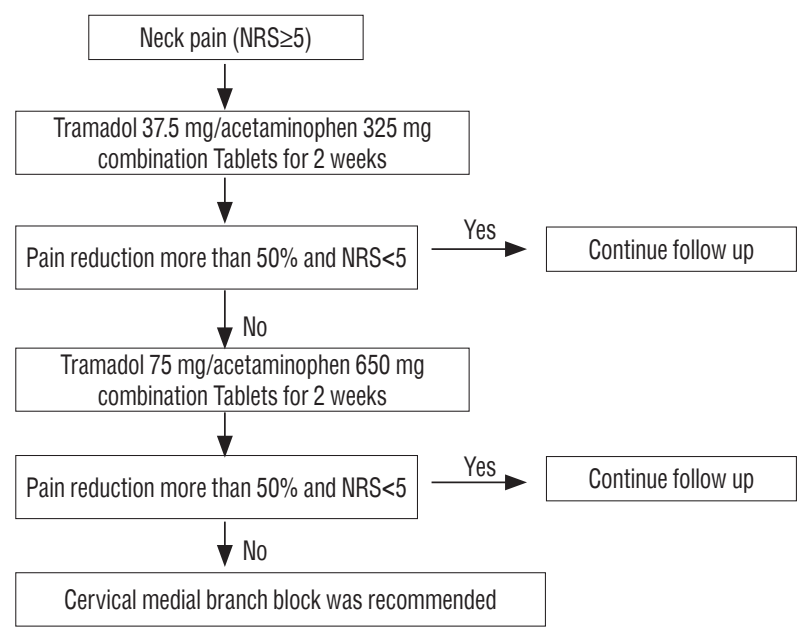

Fig. 1. Management protocol for chronic neck pain. NRS : numeric rating scale. 


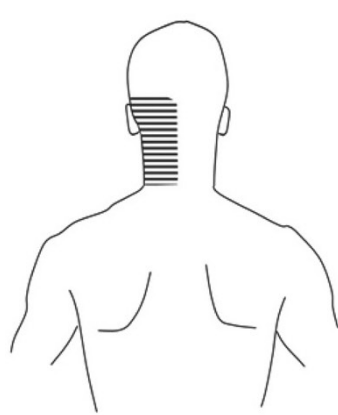

Type I

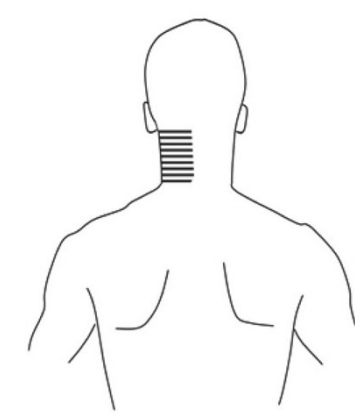

Type II

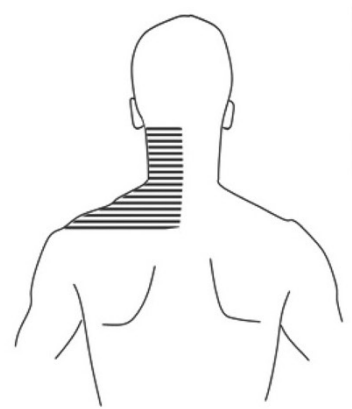

Type III

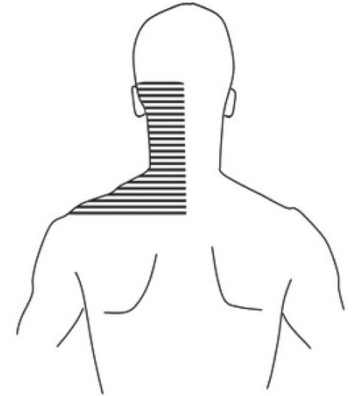

Type IV

Fig. 2. Patients were classified into four subtypes according to the location of pain. Lower occipital and neck pain was classified as Type I; isolated neck pain as Type II; neck and upper shoulder pain as Type III, lower occipital, neck, and shoulder pain as Type IV.

joint is often associated with referred neck pain ${ }^{5,13,19)}$. For diagnostic purposes, we selected to evaluate three levels, from proximal to distal facet joints, according to similar somatic referred pain territories. MBB was performed at levels C-3, C-4, and C-5 in Type I patients, at levels C-4, C-5, and C-6 levels in Type II patients, at levels C-5, C-6, and C-7 in Type III patients, and at levels C-3, C-4, C-5, C-6, and C-7 in Type IV patients. In Type I, II, and III patients whose pain did not improve, MBB at levels C-3, C-4, C-5, C-6, and C-7 was performed again the following day. A single pain specialist performed all MBB procedures, utilizing a lateral approach with the patient in the supine position. Each MBB was performed with a 22-gauge, 2 -inch spinal needle. The target points for medial branches were identified at the midpoint of the lateral mass of the cervical spine under fluoroscopic lateral visualization. After bone touch of needle and contrast injection, MBB was performed utilizing a mixture of $1 \mathrm{~mL}$ of $0.375 \%$ levobupivacaine and triamcinolone $(5 \mathrm{mg})$ at each level.

Following MBB, patients were directed to take a combination of tramadol (37.5 mg) and acetaminophen $(325 \mathrm{mg}$ ) orally, as needed (p.r.n.). In patients undergoing the MBB procedure, we evaluated changes in NRS, neck duality index (NDI), and the physical and mental component summary (PCS and MCS) of SF-36, in an outpatient clinic at 1 month post-procedure.

All patients were evaluated at 1, 3, 6, and 12 months at the outpatient clinic (minimal follow-up was 1 year). Patients who did not visit the outpatient clinic until 12 months post-procedure were regarded as lost to follow-up. Although cervical Xrays including anterior-posterior, lateral, flexion, and extension images were recommended for all study patients, a subset of patients refused this procedure. Lateral X-rays were used to differentiate the curvature classification of the cervical spine into the following subtypes : lordotic, kyphotic, straight, and sigmoid curvatures (Fig. 3).

Lateral X-ray images were evaluated for the cervical degeneration index to quantitatively and qualitatively assess the extent of degeneration. Spinal degeneration was classified into four grades according to 4 individual factors, including disc height loss, the severity of osteophytes, the amount of olisthesis, and endplate sclerosis from C2-3 through C6-7, based on a four-point scale, ranging from 0 to 3 . For each factor, a normal appearance $=0$, and the maximally severe degenerative changes $=3$ at each spinal level. The overall degree of degeneration was calculated as the sum of above factors for all levels ${ }^{36)}$. Since the degeneration score for each level ranged from 0 to 12 (with a total score ranging from 0 to 60), we classified Grade I (i.e., most normal) as a score from 0 to 20, Grade II from 21 to 40, and Grade III (i.e., the most spondylotic) from 41 to 60.

We analyzed the success rate using our treatment protocol for management of neck pain.

All patients were classified into two groups according to pharmacological treatment success or failure (i.e., mild neck pain vs. severe neck pain). Patients whose pain was successfully controlled with pharmacological treatment alone were stratified into the mild neck pain group while patients requiring cervical MBB were stratified into the severe neck pain group. Five patients in the severe neck pain group who were unable to receive medicine were excluded from the statistical analysis. Logistic regression analysis was used to elucidate the specific factors affecting the severity of neck pain. Since lateral 


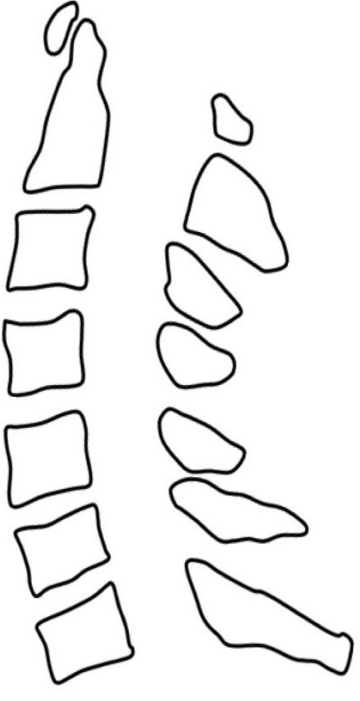

Lordotic

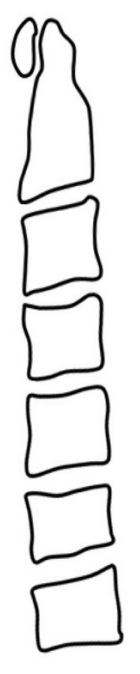

Straight
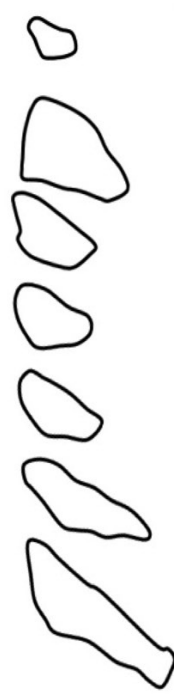

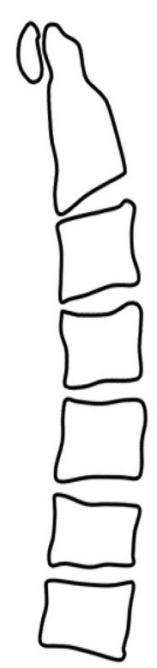

0

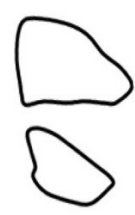

$\square$

0

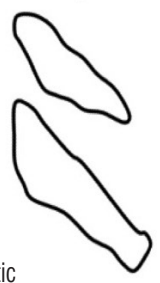

Kyphotic
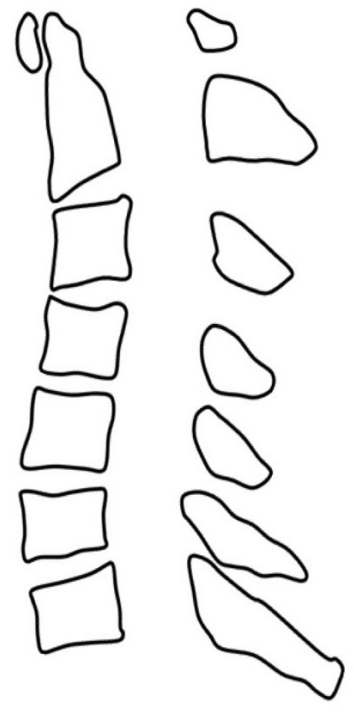

Sigmoid

Fig. 3. Cervical curvature classifications.

X-ray imaging was not possible in all patients, statistical analyses were performed twice, including patients who were not performed X-ray lateral image or not including.

\section{RESULTS}

Eighteen patients were lost to follow-up during the first year. Data from the remaining 198 patients ( 99 males and 99 females) were analyzed in this study (mean age $=57$ years, age range $=21-82$ years). Fifteen patients $(7.6 \%)$ presented with an initial history related to whiplash-associated disorder. Patients were classified with Type I ( $\mathrm{n}=22)$, Type II ( $\mathrm{n}=52)$, Type III, $(\mathrm{n}=94)$ or Type IV $(\mathrm{n}=30)$ neck pain. The initial mean NRS score was 8.0 (range $=5-10)$.

Lateral X-ray studies, performed on 165 patients, revealed the presence of 37 (22.4\%) sigmoid, 8 (4.8\%) kyphotic, 7 (4.2\%) straight, and 113 (68.5\%) lordotic curvatures. With respect to the extent of spinal degeneration, 143 patients (86.7\%) were classified as Grade I, 18 patients (10.9\%) were classified as Grade II, and 4 patients (2.4\%) as Grade III.

Fig. 4 presents the patient response to pharmacological treatment. Treatment with oral medication was successful in 135 patients (68.2\%). Tramadol $(37.5 \mathrm{mg})$ and acetaminophen $(325 \mathrm{mg})$ were successfully used to treat 131 patients and com-

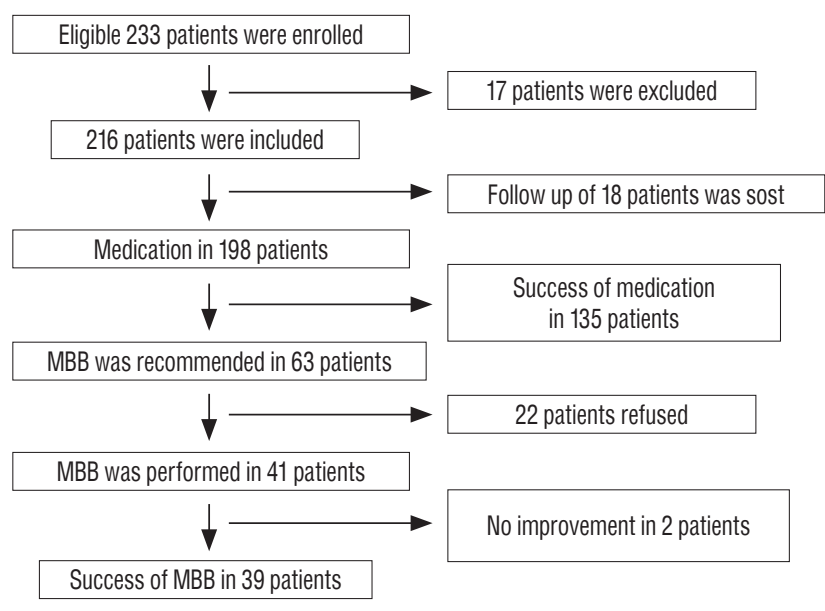

Fig. 4. Detailed result of our treatment protocol in patients. MBB : medial branch block.

bined acetaminophen $(250 \mathrm{mg})$, ibuprofen $(200 \mathrm{mg})$ and codeine phosphate $(10 \mathrm{mg})$ was successfully used in 4 patients who had complained of side effects from the original treatment paradigm. Only one patient was successfully treated with combined tramadol $(75 \mathrm{mg})$ and acetaminophen $(650$ $\mathrm{mg}$ ). This population included 69 male and 66 female subjects, with a mean age of 50.4 years (range $=21-80$ years). Nine patients $(6.7 \%)$ reported an initial history related to whiplash-associated disorder. Patients were classified with Type I ( $n=13)$, Type II ( $n=41)$, Type III, $(n=62)$ or Type IV $(n=19)$ neck pain. 

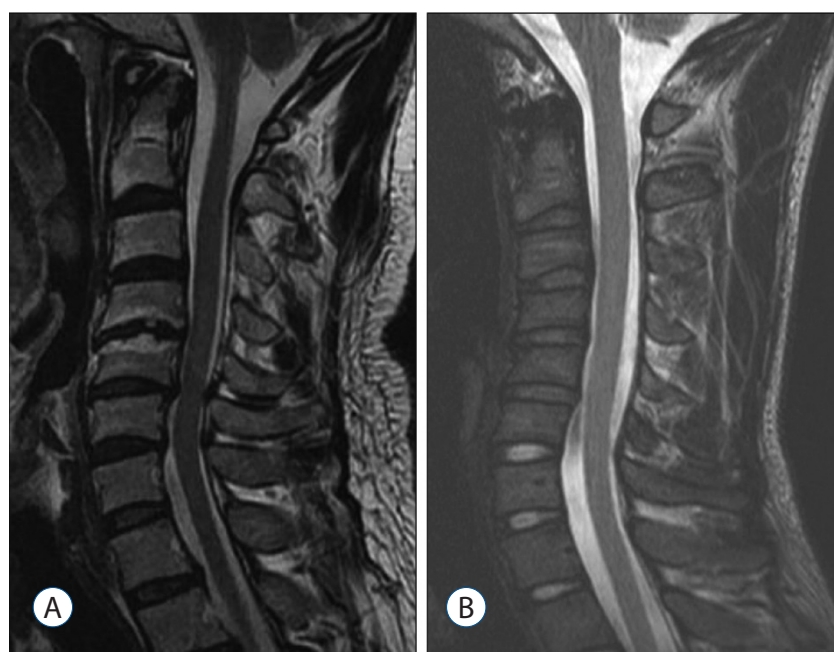

Fig. 5. A : MRI of 44-year-old male patients. MRI showed degenerative dark signals in multiple disc spaces and degenerative changes at the C4-5 level. B : MRI of 23-year-old male patients. MRI showed degenerative dark signals in multiple disc spaces. MRI : magnetic resonance imaging.

Following pharmacological treatment, the mean NRS improved from $7.0 \pm 1.00$ to $2.0 \pm 1.28$. X-ray follow-up, performed in 112 patients, revealed the presence of $20(17.9 \%)$ sigmoid, 5 (4.5\%) kyphotic, 3 (2.7\%) straight, and 84 (75.0\%) lordotic curvatures. With respect to the extent of spinal degeneration, 99 patients (88.4\%) were classified as Grade I, 11 patients $(9.8 \%)$ were classified as Grade II, and 2 patients (1.8\%) were classified as Grade III.

Sixty-three patients (30 males and 33 females) required cervical MBB. Five patients were included due to gastrointestinal trouble. The mean age for this subgroup was 57.0 years (range $=23-82$ years), and 6 patients $(9.5 \%)$ presented with an initial history related to whiplash-associated disorder. Patients were classified with Type I ( $\mathrm{n}=9)$, Type II ( $\mathrm{n}=11)$, Type III $(\mathrm{n}=32)$ or Type IV $(\mathrm{n}=11)$ neck pain. The initial mean NRS score was 8.0 (range $=5-10)$. X-ray follow-up, performed in 53 patients, revealed the presence of 17 (32.1\%) sigmoid, 3 (5.7\%) kyphotic, 4 (7.5\%) straight, and 29 (54.7\%) lordotic curvatures. With respect to the extent of spinal degeneration, $44 \mathrm{pa}-$ tients (83.0\%) were classified as Grade I, 7 patients (13.2\%) were classified as Grade II, and 2 patients (3.8\%) as Grade III. Among above 63 patients, 22 of the 63 patients declined the cervical MBB procedure, resulting in a study population of 49 patients who received the procedure. Following MBB, 39 patients (95.1\%) showed an improvement of mean NRS of neck pain (from 8.0 \pm 0.01 to $2.25 \pm 1.04$ ). The mean PCS and MCS of
SF-36 similarly improved from $33.6 \pm 5.44$ and $33.8 \pm 5.65$ to $46.5 \pm 6.10$ and $41.4 \pm 5.72$, respectively. The mean NDI score also improved from $27.0 \pm 8.15$ to $7.8 \pm 4.22$. Among the $39 \mathrm{pa}$ tients who showed clinical improvement, 10 patients (25.6\%) required repeated $\mathrm{MBB}$ due to recurrent neck pain after a minimum of 3 months. Twenty-nine patients (74.4\%) didn't show any complaints regarding recurrent symptoms.

Forty-four and 23 male patients who failed to show any clinical improvement despite cervical MBB showed a sigmoid type curvature on X-ray lateral image. The severity of neck pain in these patients was classified as Type IV with Grade I spinal degeneration. The twenty-three male patients showed a history of whiplash-associated injury. Cervical magnetic resonance imaging (MRI), performed on these two patients, revealed degenerative dark signals localized to multiple disc spaces (Fig. 5). Tables 1 and 2 present the lateral cervical curvatures including straight and sigmoid curvature significantly associated with the severity of neck pain.

\section{DISCUSSION}

Recurring neck pain is well known to disturb daily living and its natural course and prognosis have been extensively documented ${ }^{7,11,24,38)}$. Dysfunctional cervical intervertebral discs, cervical facet joints, atlanto-axial and atlanto-occipital joints, ligaments, fascia, muscles, and nerve root dura have been shown to induce pain in the cervical spine with the resulting sequelae of neck pain, upper extremity pain, and headache. In the absence of other organic causes, pain medication is routinely recommended for patients suffering from this widespread disorder.

Cervical MBB is traditionally recommended for patients with severe, uncontrolled neck pain not alleviated by medication. Based on studies involving controlled diagnostic blocks, cervical facet joints have been implicated as an underlying factor precipitating pain in the neck, head, and upper extremities in $36 \%$ to $67 \%$ of patients ${ }^{15,17}$. Although published reviews have documented evidence for efficacy of cervical MBB, a significant controversy surrounds the various treatments used in the management of chronic neck pain arising from cervical facet joints ${ }^{16,17,33)}$. One recent review reported that the evidence level for therapeutic cervical MBB and radiofrequency neurotomy of medial branches in the cervical spine was moderate, 
Prognostic Factor for Chronic Neck Pain I Seong HY, et al.

Table 1. Logistic regression analysis including patients who did not receive a lateral $\mathrm{X}$-ray imaging study

\begin{tabular}{|c|c|c|c|c|}
\hline \multirow{2}{*}{$\begin{array}{r}\text { Affecting } \\
\text { factors }\end{array}$} & \multicolumn{2}{|c|}{ Univariate analysis } & \multicolumn{2}{|c|}{ Multivariate analysis } \\
\hline & Odds ratio & $p$-value & Odds ratio & $p$-value \\
\hline Age & 1.015 & 0.188 & 1.023 & 0.093 \\
\hline \multicolumn{5}{|l|}{ Sex } \\
\hline Male & 0.957 & 0.887 & 0.835 & 0.605 \\
\hline Female & 1 & & 1 & \\
\hline \multicolumn{5}{|l|}{ WAD } \\
\hline Yes & 1 & & 1 & \\
\hline No & 0.619 & 0.385 & 0.827 & 0.760 \\
\hline \multicolumn{5}{|l|}{ Curvature } \\
\hline Kyphotic & 2.100 & 0.979 & 1.781 & 0.470 \\
\hline Straight & 4.667 & 0.220 & 5.501 & $0.044^{*}$ \\
\hline Sigmoid & 2.975 & 0.343 & 3.205 & $0.006^{*}$ \\
\hline Lordotic & 1 & & 1 & \\
\hline Unknown $^{\dagger}$ & 1.522 & 0.371 & 2.070 & 0.124 \\
\hline \multicolumn{5}{|l|}{ Degeneration } \\
\hline 0 & 1 & & 1 & \\
\hline 1 & 1.575 & 0.580 & 1.286 & 0.681 \\
\hline 2 & 1.238 & 0.976 & 0.799 & 0.870 \\
\hline Unknown ${ }^{\dagger}$ & 1.076 & 0.798 & \multicolumn{2}{|c|}{ Not applicable } \\
\hline \multicolumn{5}{|l|}{ Type } \\
\hline I & 1 & & 1 & \\
\hline$\|$ & 0.498 & 0.119 & 0.404 & 0.145 \\
\hline III & 0.929 & 0.533 & 0.779 & 0.649 \\
\hline IV & 0.880 & 0.777 & 0.725 & 0.622 \\
\hline
\end{tabular}

A total of 193 patients were evaluated, following the exclusion of five patients who refused drug treatment due to side effects, $n=58$, represents the number of patients whose pain symptoms were unrelieved with pharmacological therapy. *Means statistically significant, 'Patients who did not receive lateral X-ray imaging studies, "We were unable to estimate statistical values because two identical groups were classified a unknown with respect to curvature and degeneration. WAD: whiplash-associated disorder

or level II-1 ${ }^{17,31)}$. Cervical MBB for the management of chronic neck pain continues to be one of the most commonly performed interventions and is recognized to be safer and easier than other cervical epidural procedures ${ }^{4)}$.

Little is known, to date, regarding the success of management of chronic neck pain, including pharmacological therapy and MBB. The goal of the present study was to characterize the proportion of patients with chronic neck pain who could be successfully managed with either treatment strategy. According
Table 2. Logistic regression analysis following the exclusion of patients who did not receive $\mathrm{X}$-ray lateral imaging

\begin{tabular}{|c|c|c|c|c|}
\hline \multirow{2}{*}{$\begin{array}{r}\text { Affecting } \\
\text { factors }\end{array}$} & \multicolumn{2}{|c|}{ Univariate analysis } & \multicolumn{2}{|c|}{ Multivariate analysis } \\
\hline & Odds ratio & $p$-value & Odds ratio & $p$-value \\
\hline Age & 1.009 & 0.457 & 1.014 & 0.349 \\
\hline \multicolumn{5}{|l|}{ Sex } \\
\hline Male & 1.012 & 0.973 & 0.810 & 0.585 \\
\hline Female & 1 & & 1 & \\
\hline \multicolumn{5}{|l|}{ WAD } \\
\hline Yes & 1 & & 1 & \\
\hline No & 0.538 & 0.278 & 0.720 & 0.606 \\
\hline \multicolumn{5}{|l|}{ Curvature } \\
\hline Kyphotic & 2.100 & 0.333 & 1.898 & 0.420 \\
\hline Straight & 4.667 & 0.054 & 5.469 & $0.043^{*}$ \\
\hline Sigmoid & 2.975 & 0.007 & 2.980 & $0.011^{*}$ \\
\hline Lordotic & 1 & & 1 & \\
\hline \multicolumn{5}{|c|}{ Degeneration } \\
\hline 0 & 1 & & 1 & \\
\hline 1 & 1.575 & 0.381 & 1.406 & 0.587 \\
\hline 2 & 1.238 & 0.863 & 0.846 & 0.903 \\
\hline \multicolumn{5}{|l|}{ Type } \\
\hline । & 1 & & 1 & \\
\hline$\|$ & 0.516 & 0.300 & 0.470 & 0.267 \\
\hline III & 0.926 & 0.890 & 0.808 & 0.718 \\
\hline IV & 1.200 & 0.780 & 1.113 & 0.875 \\
\hline
\end{tabular}

A total of 160 patients were evaluated following the exclusion of five patients who refused drug treatment due to side effects, $n=48$, represents the number of patients whose pain symptoms were unrelieved by pharmacological therapy. *Means statistically significant. WAD : whiplash-associated disorder

to the World Health Organization pain ladder, a combination of non-steroidal anti-inflammatory drugs, acetaminophen and "weak" narcotics is recommended for treatment of moderate pain (NRS of 5-7 $)^{29,37)}$. Our initial drug treatment regimen was based on this recommendation. Our results show that medication is successful in $68.2 \%$ of patients over a short period. It is possible that the 18 patients who were lost during follow-up may have responded poorly to the medication regimen. If we incorporate the lost patients into the medication failure category, our success rate with drug treatment alone was $62.5 \%$ (135/216). Patients were advised to self-administered medication p.r.n. and encouraged to perform neck exercises when lower neck pain returned. Our results show that a lower dose of med- 
ication was sufficient to reduce pain in all but one patients responding to an elevated dose of medication. Cervical MBB was recommended for the remaining patients who were unresponsive to the elevated dose drug regimen and continued to suffer from sustained neck pain (NRS $>5$ ). Previous studies have reported neck pain with shoulder area involvement ${ }^{1,8,20,40,41)}$. The referred pain of the lower cervical spinal zygaphophyseal joint is also known to involve the shoulder, ${ }^{5,13,19}$. Our study showed that the highest prevalence of simultaneous neck and shoulder involvement, thus we performed MBB at levels C6-7 in Type III or IV patients.

Spinal degenerative changes often begin in the intervertebral disc space, leading to changes in the surrounding bony anatomy and soft tissues. Cervical spondylosis is a generic term for the degenerative cascade that may affect the entire cervical spine and may be seen radiographically in both symptomatic and asymptomatic individuals suggesting that degeneration progression may be unrelated to the presence of neck pain ${ }^{9}$. Although a lack of consensus currently exists, several studies regarding the association between cervical degeneration and neck pain have been reported ${ }^{27,34,39)}$. Although we analyzed progressive cervical degeneration and its relation to the severity of neck pain in the present study, the extent of degeneration was not also related with the severity of neck pain in our result.

Recent clinical studies have attempted to elucidate the association between cervical alignment and axial symptoms. However, the extent and nature of this relationship remains inconclusive. Although several studies have reported that cervical alignment is unrelated to cervical degeneration or neck pain in non-operative patients, one study has suggested that cervical kyphotic fusion may be associated with axial neck pain $^{21,22,26)}$. The results of the present study demonstrate that lateral cervical curvature is significantly associated with the severity of neck pain.

Our study has several limitations. First, even though our patients showed no evidence of radiculopathy and/or myelopathy, we did not perform a cervical MRI in all patients and therefore were unable to diagnose the patient with discogenic neck pain. In addition, we performed cervical MBB only when pharmacological pain treatment failed; many procedures exist to relieve pain, such as epidural block, facet block, and tender point injection. Moreover, the short follow-up duration and small number of patients prevent us from defini- tively elucidating the factors underlying chronic neck pain.

\section{CONCLUSION}

We successfully treated chronic neck pain with a simple pharmacological management protocol. Pain medication was successful in $68.2 \%$ of patients, while the remaining patients required cervical MBB. Among the patients who received cervical MBB, 39 (95.1\%) reported successful relief from neck pain. Although ten patients required repeated $\mathrm{MBB}$, the remaining 29 patients required only a single MBB procedure during the 1-year study period. Lateral cervical curvature was found to be significantly associated with the severity of neck pain. We should keep in mind that it may be difficult to manage the patient with straight or sigmoid lateral curvature only with oral medication.

\section{References}

1. Alperovitch-Najenson D, Sheffer $D$, Treger I, Finkels $T$, Kalichman $L$ : Rehabilitation versus nursing home nurses' low back and neck-shoulder complaints. Rehabil Nurs $40: 286-293,2015$

2. American Society of Anesthesiologists Task Force on Chronic Pain Management, American Society of Regional Anesthesia and Pain Medicine Practice guidelines for chronic pain management: an updated report by the American Society of Anesthesiologists Task Force on Chronic Pain Management and the American Society of Regional Anesthesia and Pain Medicine. Anesthesiology 112 : 810-833, 2010

3. Barnsley $L$ : Percutaneous radiofrequency neurotomy for chronic neck pain: outcomes in a series of consecutive patients. Pain Med 6 : 282286, 2005

4. Benyamin RM, Datta S, Falco FJ : A perfect storm in interventional pain management: regulated, but unbalanced. Pain Physician 13 : 109116, 2010

5. Bogduk N : Practice guidelines: spinal diagnostic and treatment procedures. San Francisco : International Spine Intervention Society, 2004

6. Borghouts JA, Koes BW, Bouter LM : The clinical course and prognostic factors of non-specific neck pain: a systematic review. Pain $77: 1-13$, 1998

7. Bot SD, van der Waal JM, Terwee CB, van der Windt DA, Scholten RJ, Bouter LM, et al. : Predictors of outcome in neck and shoulder symptoms: a cohort study in general practice. Spine (Phila Pa 1976) 30 : E459-E470, 2005

8. Brandt M, Sundstrup E, Jakobsen MD, Jay K, Colado JC, Wang Y, et al. : 
Association between neck/shoulder pain and trapezius muscle tenderness in office workers. Pain Res Treat 2014 : 352735, 2014

9. Buckwalter JA : Aging and degeneration of the human intervertebral disc. Spine (Phila Pa 1976) 20 : 1307-1314, 1995

10. Carroll LJ, Hogg-Johnson S, van der Velde G, Haldeman S, Holm LW, Carragee EJ, et al. : Course and prognostic factors for neck pain in the general population: results of the Bone and Joint Decade 2000-2010 Task Force on Neck Pain and Its Associated Disorders. Spine (Phila Pa 1976) 33(4 Suppl) : S75-S82, 2008

11. Côté $P$, Cassidy JD, Carroll LJ, Kristman V : The annual incidence and course of neck pain in the general population: a population-based cohort study. Pain 112 : 267-273, 2004

12. Croft PR, Lewis M, Papageorgiou AC, Thomas E, Jayson MI, Macfarlane GJ, et al. : Risk factors for neck pain: a longitudinal study in the general population. Pain 93 : 317-325, 2001

13. Dwyer A, Aprill C, Bogduk N : Cervical zygapophyseal joint pain patterns. I : a study in normal volunteers. Spine (Phila Pa 1976) 15 : 453-457, 1990

14. Enthoven P, Skargren E, Oberg B : Clinical course in patients seeking primary care for back or neck pain: a prospective 5-year follow-up of outcome and health care consumption with subgroup analysis. Spine (Phila Pa 1976) 29 : 2458-2465, 2004

15. Falco FJ, Datta S, Manchikanti L, Sehgal N, Geffert S, Singh V, et al. : An updated review of the diagnostic utility of cervical facet joint injections. Pain Physician 15 : E807-E838, 2012

16. Falco FJ, Erhart S, Wargo BW, Bryce DA, Atluri S, Datta S, et al. : Systematic review of diagnostic utility and therapeutic effectiveness of cervical facet joint interventions. Pain Physician 12 : 323-344, 2009

17. Falco FJ, Manchikanti L, Datta S, Wargo BW, Geffert S, Bryce DA, et al. : Systematic review of the therapeutic effectiveness of cervical facet joint interventions: an update. Pain Physician 15 : E839-E868, 2012

18. Fejer R, Kyvik KO, Hartvigsen $J$ : The prevalence of neck pain in the world population: a systematic critical review of the literature. Eur Spine J 15 : 834-848, 2006

19. Fukui S, Ohseto K, Shiotani M, Ohno K, Karasawa H, Naganuma Y, et al. : Referred pain distribution of the cervical zygapophyseal joints and cervical dorsal rami. Pain $68:$ 79-83, 1996

20. Ghafouri N, Ghafouri B, Fowler CJ, Larsson B, Turkina MV, Karlsson L, et al. : Effects of two different specific neck exercise interventions on palmitoylethanolamide and stearoylethanolamide concentrations in the interstitium of the trapezius muscle in women with chronic neck shoulder pain. Pain Med 15 : 1379-1389, 2014

21. Gore DR, Sepic SB, Gardner GM : Roentgenographic findings of the cervical spine in asymptomatic people. Spine (Phila Pa 1976) 11 : 521524, 1986

22. Grob D, Frauenfelder $H$, Mannion AF : The association between cervical spine curvature and neck pain. Eur Spine J 16 : 669-678, 2007

23. Hartvigsen J, Frederiksen $H_{1}$ Christensen $\mathrm{K}$ : Back and neck pain in seniors-prevalence and impact. Eur Spine J 15 : 802-806, 2006

24. Hill J, Lewis M, Papageorgiou AC, Dziedzic K, Croft $P$ : Predicting persistent neck pain: a 1-year follow-up of a population cohort. Spine (Ph- ila Pa 1976) 29 : 1648-1654, 2004

25. Hoving JL, de Vet HC, Twisk JW, Devillé WL, van der Windt D, Koes BW, et al. : Prognostic factors for neck pain in general practice. Pain 110 : 639-645, 2004

26. Kawakami M, Tamaki T, Yoshida M, Hayashi N, Ando M, Yamada H : Axial symptoms and cervical alignments after cervical anterior spinal fusion for patients with cervical myelopathy. J Spinal Disord 12 : 50-56, 1999

27. Laimi K, Pitkänen J, Metsähonkala L, Vahlberg T, Mikkelsson M, Erkintalo $\mathrm{M}$, et al. : Adolescent cervical disc degeneration in MRI does not predict adult headache or neck pain: a 5-year follow-up of adolescents with and without headache. Cephalalgia 34 : 679-685, 2014

28. Lord SM, Barnsley L, Wallis BJ, McDonald GJ, Bogduk N : Percutaneous radio-frequency neurotomy for chronic cervical zygapophyseal-joint pain. N Engl J Med 335 : 1721-1726, 1996

29. Malanga GA, Patel $A D$ : The pharmacologic management of spine pain in Rao RD, Smuck M (eds) : Orthopaedic knowledge update : spine, ed 4. Rosemont, IL : American Academy of Orthopaedic Surgeons, 2012, pp93-105

30. Manchikanti L, Boswell MV, Singh V, Benyamin RM, Fellows B, Abdi $S$, et al. : Comprehensive evidence-based guidelines for interventional techniques in the management of chronic spinal pain. Pain Physician $12: 699-802,2009$

31. Manchikanti L, Manchikanti KN, Damron KS, Pampati V : Effectiveness of cervical medial branch blocks in chronic neck pain: a prospective outcome study. Pain Physician 7 : 195-201, 2004

32. Manchikanti L, Singh V, Falco FJ, Cash KA, Fellows B : Comparative outcomes of a 2-year follow-up of cervical medial branch blocks in management of chronic neck pain: a randomized, double-blind controlled trial. Pain Physician 13 : 437-450, 2010

33. Manchikanti L, Singh V, Helm S 2nd, Trescot AM, Hirsch JA : A critical appraisal of 2007 American College of Occupational and Environmental Medicine (ACOEM) Practice Guidelines for Interventional Pain Management: an independent review utilizing AGREE, AMA, IOM, and other criteria. Pain Physician 11 : 291-310, 2008

34. Marchiori DM, Henderson CN : A cross-sectional study correlating cervical radiographic degenerative findings to pain and disability. Spine (Phila Pa 1976) 21 : 2747-2751, 1996

35. Martin BI, Deyo RA, Mirza SK, Turner JA, Comstock BA, Hollingworth W, et al. : Expenditures and health status among adults with back and neck problems. JAMA 299 : 656-664, 2008

36. Ofiram E, Garvey TA, Schwender JD, Denis F, Perra JH, Transfeldt EE, et al. : Cervical degenerative index: a new quantitative radiographic scoring system for cervical spondylosis with interobserver and intraobserver reliability testing. J Orthop Traumatol $10: 21-26,2009$

37. Park JH, Jeong EK, Lee MK, Chul Rhim S, Roh SW, Kim JH, et al. : A unilateral open door laminoplasty technique: prospective analysis of the relationship between midline extensor muscle preservation and postoperative neck pain. J Clin Neurosci 22 : 308-314, 2015

38. Pernold G, Mortimer M, Wiktorin C, Tornqvist EW, Vingård E; Musculoskeletal Intervention Center-Norrtälje Study Group : Neck/shoulder 
J Korean Neurosurg Soc 60 | July 2017

disorders in a general population. Natural course and influence of physical exercise: a 5-year follow-up. Spine (Phila Pa 1976) 30 : E363E368, 2005

39. Peterson C, Bolton J, Wood AR, Humphreys BK : A cross-sectional study correlating degeneration of the cervical spine with disability and pain in United kingdom patients. Spine (Phila Pa 1976) 28 : 129-133, 2003

40. Rasmussen-Barr E, Grooten WJ, Hallqvist J, Holm LW, Skillgate E : Are job strain and sleep disturbances prognostic factors for neck/shoulder/ arm pain? A cohort study of a general population of working age in Sweden. BMJ Open 4 : e005103, 2014

41. Ruivo RM, Pezarat-Correia P, Carita Al : Cervical and shoulder postural assessment of adolescents between 15 and 17 years old and association with upper quadrant pain. Braz J Phys Ther 18 : 364-371, 2014

42. Yin $W$, Bogduk $N$ : The nature of neck pain in a private pain clinic in the United States. Pain Med 9 : 196-203, 2008 